\title{
Social wasps repair emergent topological defects
}

\author{
Shivani Krishna ${ }^{1, *}$, Apoorva Gopinath ${ }^{1}$ and Somendra M. Bhattacharjee ${ }^{2}$ \\ ${ }^{1}$ Department of Biology, Ashoka University, Sonepat 131029, India \\ ${ }^{2}$ Department of Physics, Ashoka University, Sonepat 131029, India \\ *Corresponding author: shivani.krishna@ashoka.edu.in
}

\begin{abstract}
Social insects have evolved a variety of architectural formations. Bees and wasps are well known for their ability to achieve compact structures by building hexagonal cells. Polistes wattii, an open nesting paper wasp species, builds planar hexagonal structures. Here, using the pair correlation function approach, we show that their nests exhibit short-range hexagonal order but no long-range order akin to amorphous materials. Hexagonal orientational order was well preserved globally. We also show the presence of emergent topological defects such as disclination pairs (pentagon-heptagon dipoles), Stone-Wales quadrupoles, and other higher-order defects and discuss how these defects were fixed in the nest, thereby restoring order. Furthermore, we suggest the possible role of such defects in shaping nesting architectures of other social insect species.

Keywords: nest architecture, dislocations, disclinations, paper wasps, short-range order, Burgers vector
\end{abstract}

\section{Introduction}

Animal nests and their architecture are crucial for the development of the young, protection from predators, and storage of resources. The diversity and complexity in architecture signify how these functions are fulfilled in diverse habitats and environmental conditions. Such complexity is exemplified by the nests of social insects (ants, bees,termites and wasps) owing to their ability to use or modify the surroundings, and create ordered and plastic structures (Camazine et al. 2001, Tschinkel 2004, Reid et al. 2015). These nests have captivated mathematicians and ecologists alike for their structural complexity and the mechanisms behind the coordinated building (Grassé 1984, Theraulaz et al. 1999, Hansell and Hansell 2005, Peters et al. 2019). How do multiple individuals in a group or a colony come together to construct these tiled layouts? The principle of stigmergy explains this as the formation of patterns by emergence, (Grassé 1959) including self-organisation and self-assembly mechanisms (Theraulaz and Bonabeau 1995, Bonabeau et al. 1997). In most species, the construction rules depend on environmental (e.g., humidity, soil moisture) or pheromone gradients (Camazine et al. 2001, Khuong et al. 2016). In this context, the relative importance of self-organised (Pénzes and Karsai 1993, Karsai 1999) vs template-based interactions has been examined, and current evidence points towards architectural mechanisms being an interactive effect of both these interactions (Perna and Theraulaz 2017). It is paramount to place these mechanisms in the context of behavioural and cognitive processes to understand nest construction comprehensively. The repertoires required for complex nest architectures are often characterised by simplified clusters of direct steps. However, the processes involved in planning construction in various environmental conditions, detecting errors and their remediation, working around novel obstructions, etc., require substantial cognitive abilities that outwit simple algorithms (Gallo and Chittka 2018). 
In bees and wasps, cells within the nest are usually circular or hexagonal. This preference to build hexagonal-shaped cells is attributed to compactness. Hexagonal tiling is proven to cover a planar region with regular units of equal area whilst minimising the total perimeter (honeycomb conjecture, Hales 2001). Such optimal utilisation of space and energy has been proposed as the significant selection pressure for shaping these structures (Tóth 1964, Jeanne 1975, Karsai and Pénzes 1999, Gallo and Chittka 2018). Honeybees use wax to shape their cells, while most wasp species use either mud or fibrous materials of plant origin for construction. Though the cells are hexagonal in both cases, the construction materials possess strikingly distinct properties. Therefore, the underlying processes could be different. Construction of bees' wax-based cells is postulated to begin as an array of circles (laid over packed cylinders as a base) that are modified to rounded hexagons purely by mechanical/thermodynamic means (Pirk et al. 2004, Karihaloo et al. 2013). However, experimental work from nests of European honeybees suggests that bees actively construct hexagonal cells by handling the wax and controlling its temperature (Bauer and Bienefeld 2013). Given the plasticity of wax, any disruptions to tiling in these structures can be rectified, thereby reducing the possibility of overall defects in the structure. On the other hand, nests made up of plant fibres such as those of paper wasps are unlikely to follow similar modes of construction. The reduced plasticity makes them interesting as ordered physical systems prone to topological defects. A topological defect in systems of broken symmetry, like crystals, magnets, liquid crystals, etc., is defined as a disruption of order throughout the system in a way that defies restoration via any continuous deformation (Mermin 1979, Bhattacharjee 2017. In contrast, defects such as vacancies, hilly terrain in a flat land, etc., are geometric defects as they affect order locally in their neighbourhood without any signature far away from them. A key characteristic of topological defects is that they cannot be repaired by local rearrangements alone but instead require changes to the system globally ${ }^{1}$. Here, we refer to emergent topological defects as those that occur and affect at length scales beyond a single unit/element, such as a single cell within a nest. The emergence and evolution of such topological defects in paper wasps' nests have never been addressed.

Some of the most common topological defects in crystals and liquid crystals manifest as disclinations and dislocations (Harris 1977, de Gennes 1979). Examples of such disclinations have been shown in living systems such as protein coats of viruses (Iorio and Sen 2007) and insect corneal nanostructures (Lee et al. 2016), where insertion of pentagons into an array of packed hexagons results in gaussian curvature and breaks the sixfold rotational symmetry. Similarly, the Stone-Wales defect, characterised by two pentagons and two heptagons (Stone and Wales 1986), is a well-known defect in graphene, fullerene, and carbon nanotubes (Ma et al. 2009, Heggie et al. 2016).

Paper wasps of Polistes genus build nests hanging from one or more stalks. The nest itself is made up of open hexagonal cells. Variations in nesting architectures within paper wasps have been attributed to selection pressures such as predation from ants or flying insects, insulation from heat, and economic use of building material (Eberhard 1969, Jeanne 1975, 1979, Seeley and Heinrich 1981, Wenzel 1991). The diversity of nest forms in paper wasps and their growth have been elegantly explained by a simple set of rules by Karsai and Pénzes (1998). The model suggests that multiple forms could be built by changing the weightage assigned to different parts of the nest. However, by construction, these models describe regular hexagonal structures only. The likelihood of these models explaining changes to regular hexagonal tiling or within nest changes to order is small. In this paper, analysing the nesting architecture of paper wasps (Polistes wattii), we ask the following questions:

(a) What is the nature of ordering (i.e., short-range vs long-range) and spatial arrangement of

\footnotetext{
${ }^{1}$ Geometric defects can be amended by making changes locally at the scale of basic elements or constituents (Griffin and Spaldin 2017)
} 
cells within and between the nests? In the current context, order describes the regularity in the tiling of the cells within the nests. Short-range order (SRO) represents the arrangements of nearest neighbours in the nest, while long-range order (LRO) represents the regularity over a longer distance. More quantitatively, a nest is said to have long-range order if there is a nonzero probability of finding the corners of two cells on the same regular lattice, even for large separations between the cells.

(b) Do these nests exhibit topological defects, and whether such defects disrupt or facilitate in restoring order?

\section{Methods}

The study was conducted within the campus of Ashoka University, Sonepat, India (28. 9457 $\mathrm{N}$, $77.1021^{\circ} \mathrm{E}$ ) located at an altitude of $224 \mathrm{~m}$. The maximum temperature in summers is $45-47^{\circ}$ $\mathrm{C}$, and the lowest temperature in winters is around $4-6^{\circ} \mathrm{C}$. Wasps of the genus Polistes have a widespread distribution and are a well-studied example for the evolution of sociality and dominance hierarchies in insects. It is a primitive and speciose genus with more than 200 species (Carpenter 1996). P.wattii is known to be distributed across central and South Asia (Ceccolini 2019). They are inactive during the winter, and nest building begins once they emerge from hibernation. The construction of nests often lasts till late summer. In our study, all the analysed P.wattii nests were from within anthropogenic habitats. Nesting height was typically within the range of 5-15 m. Images of nests were taken in the summer of 2020 and 2021. Adult nests that were fully constructed and completed the season were considered for analysis (typically with atleast 50 cells, Figure S4). These nests were photographed with a reference scale. Images were further analysed by subtracting the background and binarisation using ImageJ (Schneider et al. 2012). The positions of all the individual vertices (corners shared by atleast three cells) were marked on the images, and their x, y coordinates were exported for the subsequent analysis. To avoid the effect of boundary which includes incomplete cells, a few layers of vertices along the edges were excluded. Apart from these, a few cells with cylindrical projections where identifying the underlying vertices was difficult were excluded from the final analyses. Overall, 25 nests were used to characterise the degree of ordering. The presence of topological defects was identified by inspecting the number of vertices for the cells within the nests. We characterised the defects by identifying their basic features (number of vertices and neighbouring cells) and calculating the internal angles of the first neighbouring hexagonal layer from coordinates. For a region without defects, we would expect these angles to be close to $120^{\circ}$.

To quantify ordering and analyse the spatial distribution of cells, we used the pair correlation function $g(r)$ (henceforth PCF) based on Ripley's K function derivative method in the spatstat package (Baddeley et al. 2015). The approach relies on the probability of finding a pair of vertices separated by a distance $r$. This results in an average representation of the local spatial neighbourhood at a distance $r$ from any given vertex. PCF of a regular lattice exhibits sharp peaks at the lattice spacing distances. Such sharp peaks indicate perfect ordering. On the other hand, an amorphous structure will show some reduced degree of order; in particular, it would have $g(r)$ approaching 1 for large values of $r$. The limiting value of 1 indicates no correlation between the positions of vertices when they are far apart. In a PCF, the peak heights are generally related to the number of neighbours. The first two peaks were discernible in nests of different sizes, allowing us to focus on the location and width of these peaks for each of the nests. The following function consisting of two gaussians with amplitudes $a_{1}, a_{2}$ has been used to fit the first two peaks of the $g(r)$-vs- $r$ curve for 
each nest,

$$
g(r)=a_{1} \exp \left(-\frac{\left(r-r_{1}\right)^{2}}{2 s_{1}^{2}}\right)+a_{2} \exp \left(-\frac{\left(r-r_{2}\right)^{2}}{2 s_{2}^{2}}\right)
$$

where $r_{1}, s_{1}$ represent the location and the width of the first peak, and $r_{2}, s_{2}$ of the second peak. We also calculated the coefficient of variation (CV) of location and width for the first two peaks to quantify the extent of variation in order between nests.

We measured the individual cell areas and compared the variation between and within the analysed nests. We also calculated the nearest neighbour distances or bond lengths, i.e., distances between vertices (henceforth wall lengths). Based on the the fitted values of Eq. 1, the wall length would be in the range $r_{1} \pm 2 s_{1}$. Information of translational ordering is given by the PCF, and to obtain the orientational order, we defined cell-orientational order in analogy with bond-orientational order in liquids. Cell-orientational order parameter is a measure of the geometrical arrangement of the vertices of a given cell around its cell centre (Steinhardt et al. 1983, Schilling et al. 2005). Cell-orientational order parameter $\psi_{n}$ is given by a complex number defined at each cell centre as,

$$
\psi_{n}\left(\mathbf{X}_{j}\right)=\frac{1}{N_{j}} \sum_{k=1}^{N_{j}} e^{i n \theta_{j k}},
$$

where $N_{j}$ is the number of vertices of cell $j$ centered at $\mathbf{X}_{j}, \theta_{j k}$ represents the angle between the line joining vertex $k$ to the centre and the chosen $\mathrm{x}$-axis, and $n$ is an index for orientational ordering (Figure 1). For hexagonal ordering, we choose $n=6$. Note that $\psi_{n}$ neither depends on the vertex
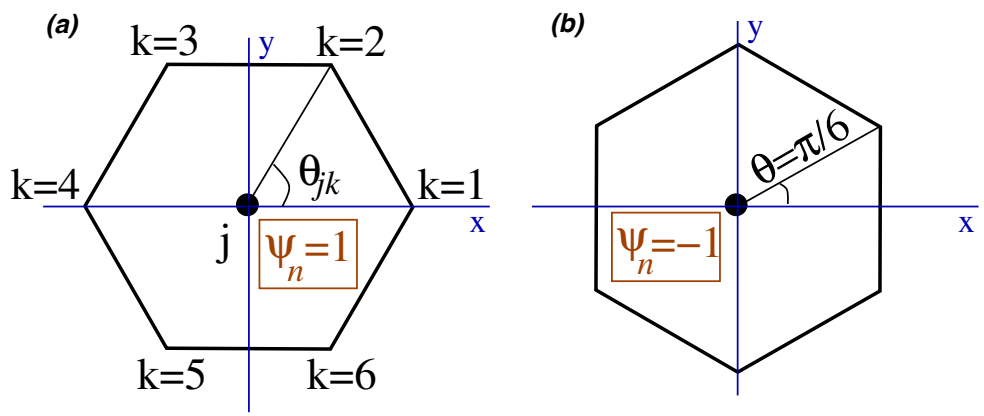

Figure 1: Cell-orientation order parameter (Eq. 2). The angle $\theta_{j k}$ is measured from an arbitrarily chosen $x$-axis through the centre as shown in (a). For a regular hexagon with inner angles at $2 \pi / 3$, the orientation in (a) has $\psi_{n}=1$ while in (b) $\psi_{n}=-1$. In general, $\psi_{n}$ is orientation-dependent with $\left|\psi_{n}\right|$ less than or equal to 1 . For regular hexagons, individual $\psi_{n}$ lies on the unit circle. The six-fold symmetry of a regular hexagon is preserved by $\psi_{n}$ as it returns to its value under a $\pi / 3$ rotation of the hexagon around its centre.

numbering scheme nor the size of the hexagon. As a complex number, $\psi_{n}=\left|\psi_{n}\right| e^{i \theta}$ lies on or within the unit circle in the complex plane, and therefore, $\psi_{n}\left(\mathbf{X}_{j}\right)$ can be represented graphically by a vector at an angle $\theta$ with the x-axis at the cell centre $\mathbf{X}_{j}$. The average $\left\langle\psi_{n}\right\rangle$ of $\psi_{n}\left(\mathbf{X}_{j}\right)$ over a large number of hexagons lies in the range $0 \leq\left|\left\langle\psi_{n}\right\rangle\right| \leq 1$ with the two extreme values representing a random orientational arrangement $\left(\left\langle\psi_{6}\right\rangle=0\right)$ and a perfect hexagonal ordering $\left(\left\langle\psi_{6}\right\rangle=1\right)$. We calculated the cell-orientational order parameter for a simulated set of random central angles to study the decay of $\left|\left\langle\psi_{6}\right\rangle\right|$ from the perfect value of 1 (see details in supplementary material). Analyses were performed by using Fortran (https://gcc.gnu.org) and R software (R Core Team 2020). 


\section{Results and Discussion}

\section{Nature of ordering and spatial arrangement of cells in the nests}

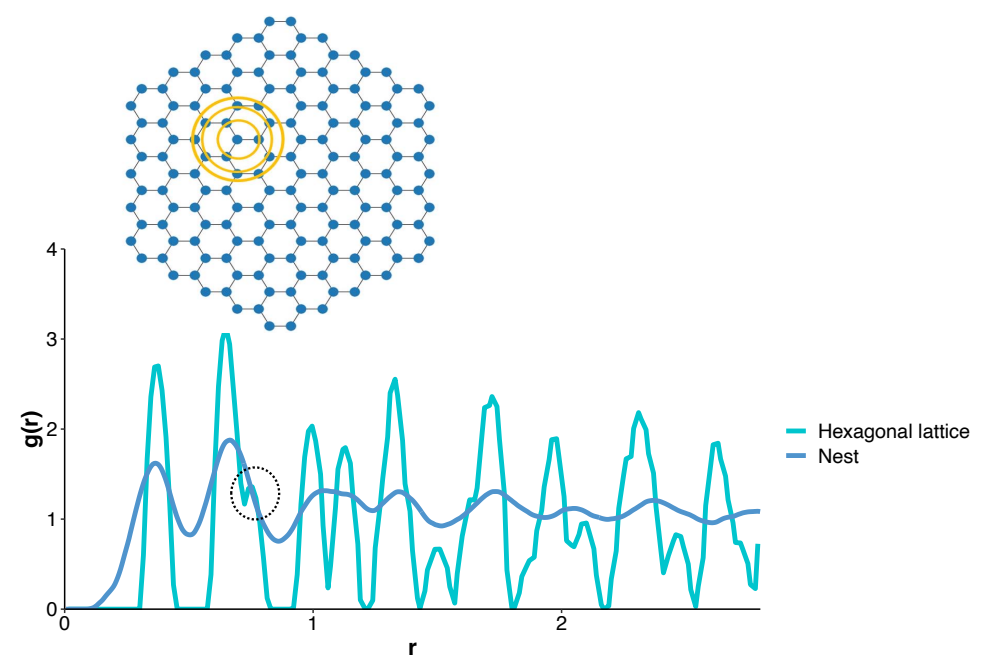

Figure 2: Pair correlation function as a function of $r$, i.e., the distance between vertices. The two curves depict a representative nest and a regular hexagonal lattice. The splitting of the second peak in a regular hexagonal lattice is indicated by a circle. A schematic on the top shows a regular hexagonal lattice and the vertices in the first, second, and third shells around any one vertex.

The number of cells in the analysed nests ranged between 50 and 740. For each nest, we characterised the nature of ordering by calculating the pair correlation function for the vertices. For a regular hexagonal lattice, sharp peaks at all distances dropping in between to zero indicate the presence of both short and long-range order (Bishop and Bruin 1984). As shown in Figure 2, for any vertex on the hexagonal lattice, the first, second, and third neighbours lie on circular shells and the first 3 PCF peaks reflect this regularity. The bond length of the regular lattice is chosen such that the first peak is at the same location as the $\mathrm{g}(r)$ of the nest. There is a substantial overlap between the first and second peaks of a regular hexagonal lattice and those of a typical nest. As $r$ increases, this overlap disappears. This pattern of the PCF illustrates the SRO in the structure of nests.

The presence of a peak confirms that any given vertex would have neighbouring vertices at distances provided by the peak position. This is an example of SRO. Moreover, as the decay of the PCF to $g(r)=1$ is not algebraic, we suggest the absence of LRO. The proximity of the third peak to the second is an inherent characteristic of a hexagonal lattice. However, this proximity is not seen in the case of nests. The absence of sharp peaks and the missing proximity (between 2nd and 3rd peak) are signatures of deviation from regularity. Indeed, the broad peaks indicate heterogeneity of cell sizes that impairs ordering over long distances. We conclude, that similar to amorphous structures, nests exhibit SRO but no LRO.

The PCF curves of nests shown in Figure 3(a) have been normalized such that the first peak begins at one (non-normalized individual nests' PCFs are shown in Figure S1). When variations in this translational order of nests were analysed, the nest-to-nest variation was found to be restricted to larger $r$ values. This is further corroborated by the low variance values (coefficient of variation, $\mathrm{CV}$ ) of location and width of the first peak compared to the second peak (Figure 3(b), (c), (d)). 

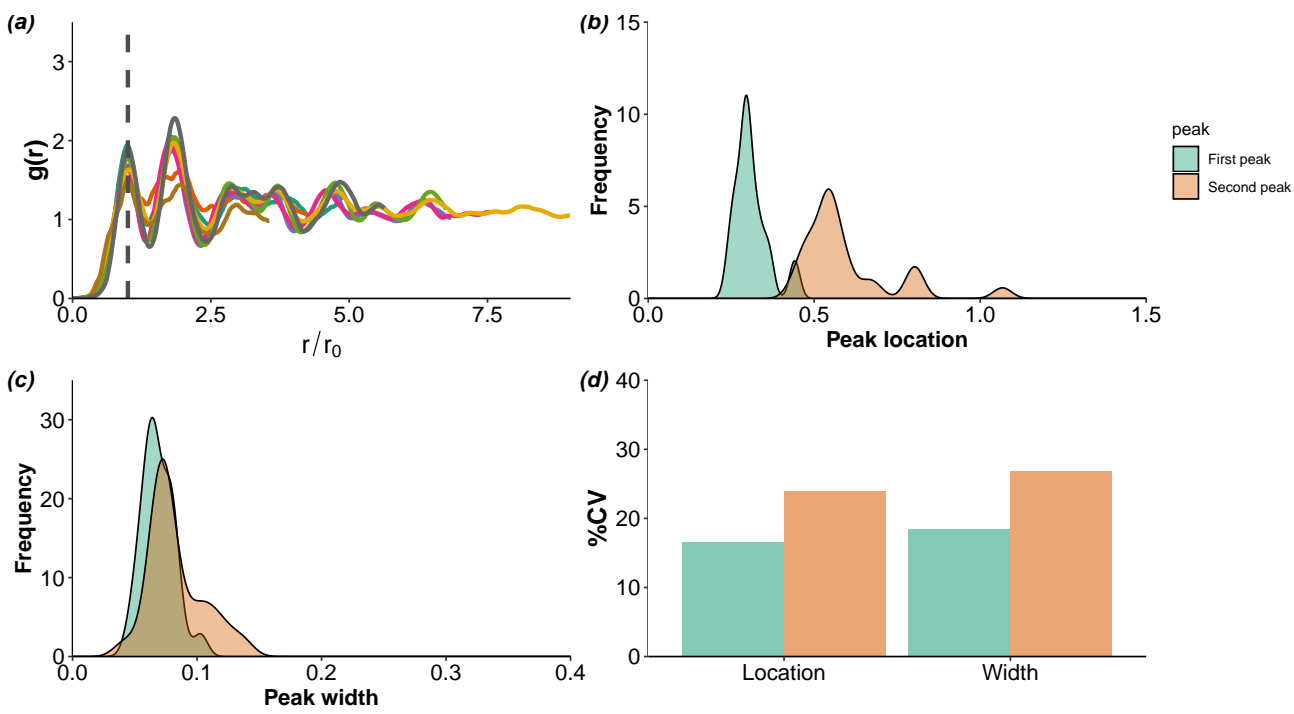

Figure 3: (a) Pair correlation function of vertices from 10 randomly chosen nests, showing a significant overlap around the first and second peaks; beyond the third peak, the positioning of vertices approaches random distribution (approaching 1$)$. The x-axis is scaled $\left(r / r_{0}\right)$ such that the first peak is at the same $\mathrm{x}$-value for all nests. Distribution of (b) locations and (c) widths obtained by gaussian approximation of first and second peaks of the pair correlation function of 25 nests (d) Relative difference in coefficient of variation (CV) of locations and widths of first and second peaks. Each CV value depicts variation within the nest.

The structural features were analysed by characterizing the distribution of wall lengths, cell areas, and cell orientational order (Figure 4). Figure 4(a) depicts the extent of variation in wall lengths between the different nests. The lengths varied within the range of 0.1 and $0.9 \mathrm{~cm}$, with a median value of $0.3 \mathrm{~cm}$. The distribution of cell wall lengths also fits well with the values obtained from the gaussian fit of the first peak of the PCF. The cell areas were in the range of 0.07 to 0.85 sq.cm (median $=0.25$ sq.cm, Figure 4(b)). Deviations from regularity can be obtained by having cells of similar areas but varying the wall lengths and vice-versa. Furthermore, we analysed if the degree of deviation was greater within the nest or between the nests. Percent CV values of cell areas ranged between 13 and 28 while the wall lengths varied from 44 to $58 \%$. The wall length variation was greater within the nests than between the nests.

Our analysis suggests that the orientation of the cells is fairly preserved and aligns well with the nearest neighbours (Figure 4(c), (d)). The order parameter varied within the range of 0.1 and 1 , with a median value of 0.85 . Significant overlap in cell orientational order parameter distributions of the nests suggests that the presence of orientational order is true across the nests (Figure 4(c)). Percent CV values varied between 16 and 31, indicating that variation within nests was smaller than the variation in wall lengths. Compared to the case of a single cell with random internal lengths (see supplementary material), the probability distribution of $\left\langle\psi_{6}\right\rangle$ in Figure 4(c) is wider. We may attribute this to the cooperative effect of building a compact nest and yet becoming amorphous-like in the large scale limit. A well-studied example of such cooperative-effect induced broadening is the probability distribution of magnetism as one approaches the Curie temperature of a magnet (Fleury 1981). Taken together, the pair correlation function and distribution of cell orientational order parameters suggest the presence of short-range hexagonal order and an overall orientational order even though there is no long-range order akin to amorphous materials. 

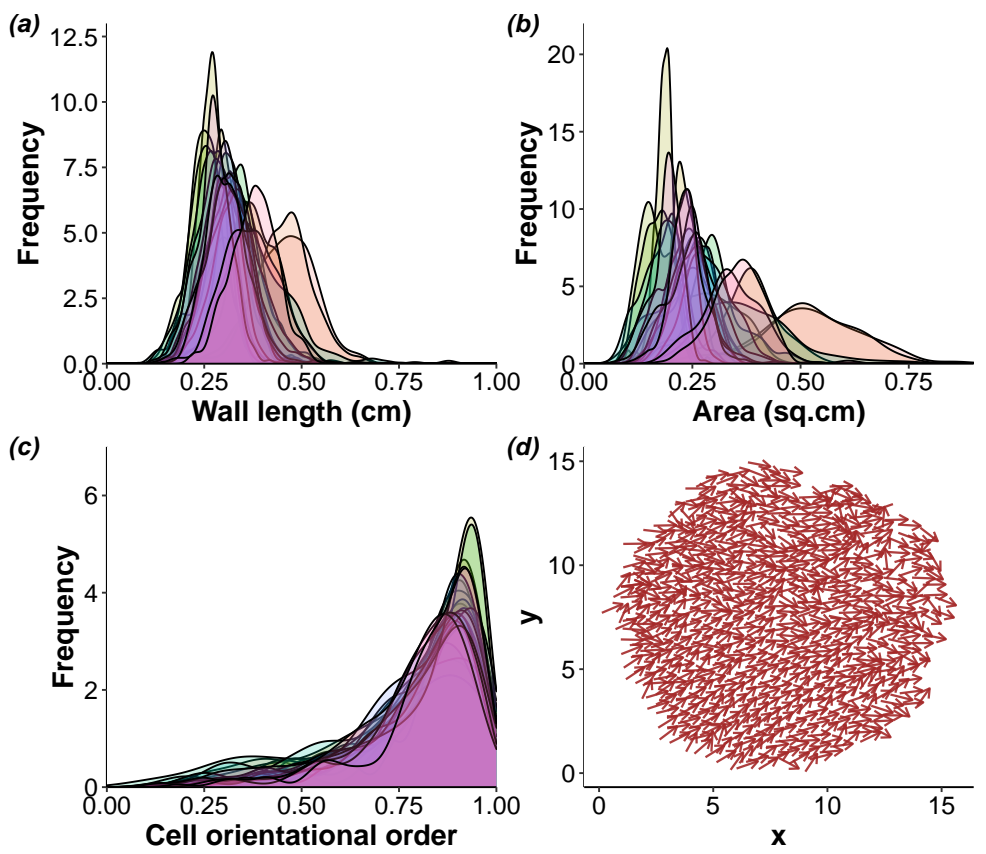

Figure 4: Frequency distribution of (a) cell wall lengths, (b) cell areas, and (c) cell orientational order parameter, depicting variation between and within the nests. (d) Quiver plot showing the direction and magnitude of cell orientational order parameter of a representative nest. Each arrow is located at the centre of the cell.

\section{Topological defects}

We detected the occurrence of non-hexagonal cells and analysed their arrangements within the nest. Figures 5 and 6 show the distinct types of such defects found within the nests. Such presence of pentagons and heptagons were reported in some nests of $P$. annularis (Wenzel 1989), where their role in the curvature of nests was suggested. However, the presence of octagons and higher-order defects has not been reported.

(a)
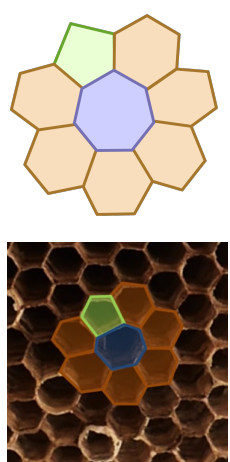

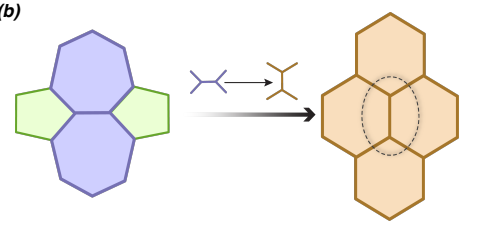

Figure 5: Schematic representation of (a) a pentagon and a heptagon amidst hexagons (dipole), and (b) Stone-Wales defect (pentagon-heptagon quadrupole).Bottom panels show their outline in wasp nests.

Existing work suggests that defects such as a) two pentagons amidst hexagons b) one pentagon or $\mathrm{c}$ ) one heptagon amidst hexagons can move the structure away from planarity as shown 
in Figure 7 (Zsoldos 2010). If structural disorders such as topological defects are left uncorrected, they could strongly affect the overall planarity of the nest. The missing link of a pentagon in a hexagonal net necessarily requires the removal of a wedge of hexagons, producing a cone-like structure as shown in Figure 7(a). Similarly, an opposite curvature can be produced by a single heptagon (Figure 7(b)) with an extra link that requires the insertion of a wedge. As these curvatures cannot be ironed out, these are topological defects called disclinations that tend to disrupt the orientational order of the lattice. A pair of the two opposite types do not cancel each other but instead carry with them a line defect, which is called a dislocation line. This dislocation line consists of an extra array of hexagonal cells (Figure 7(c)). Furthermore, by drawing a loop in a region without defects and comparing it with a similar loop that encloses a dipole, one can characterise the dislocation. The steps required to close the loop are characteristic of the defect and independent of the size and shape of the loop. This topological invariant called Burgers vector is shown in Figure $7(\mathrm{c})$.

(a)

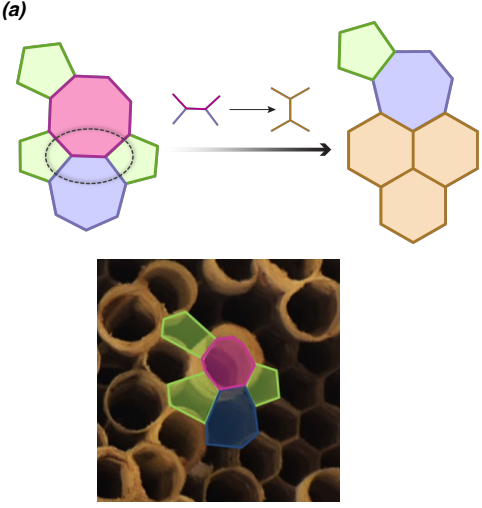

(b)

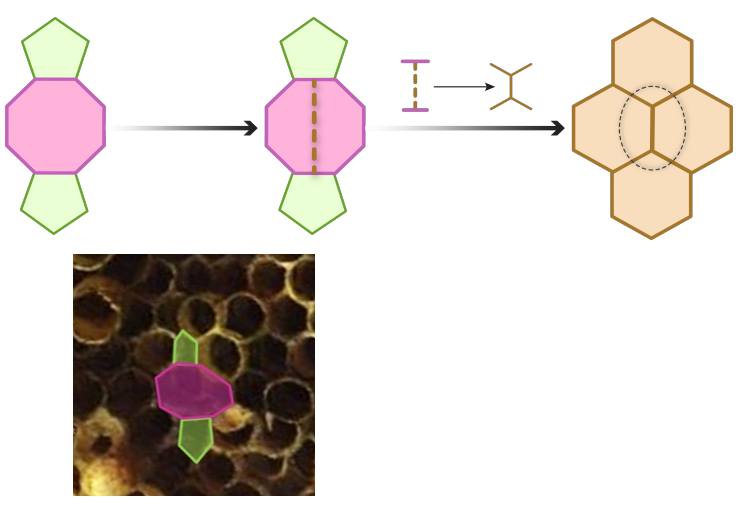

Figure 6: Schematic representation of (a) an octagon surrounded by a heptagon and three pentagons, and (b) an octagon between two pentagons. Bottom panels show their outline in wasp nests.

Stone-Wales defect comprises of two pentagons and two heptagons, which typically arises by a simple $90^{\circ}$ rotation of the bond (wall) between two hexagons (Figure 5(b)). Such topological defects are known to occur commonly in graphite and graphene (Ophus et al. 2015). For cells with defects that occur in pairs or as groups, we measured the distribution angles of the neighbouring vertices for upto one shell of nearest neighbours. For a regular hexagonal lattice, these angles would be $120^{\circ}$, and for the nest without defects, a set of randomly chosen cells subtended angles that ranged between $90^{\circ}$ and $140^{\circ}$. However, hexagonal cells adjoining the defect pairs such as pentagon-heptagon showed angles that range from $99^{\circ}$ to $151^{\circ}$, which differed both the regular hexagonal lattice as well as hexagonal cells in the nest. Figure 6(a) depicts a defect where an octagon is embedded within three pentagons, four hexagons, and a heptagon. This could originate from a $90^{\circ}$ rotation of the bond between three hexagons and a heptagon. Another defect that was observed was an octagon with two pentagons on either side (Figure 6). This can change to a four hexagon configuration by a wall addition followed by a split (change in the bond angles of the central wall). All the above-described defects were found to maintain the three-point vertices.

The most common configurations of pentagon-heptagon pairs were dipoles and the configuration where hexagons separate a pentagon and a heptagon were never observed in our dataset. Interestingly, some of the described defects and their properties are utilized by honeybees to attain curvature as well as to merge cells of different sizes (Hepburn and Whiffler 1991, Smith et al. 2021). Cells of different sizes are routinely observed in honeycombs as larvae of drones and workers are 
(a)

(b)
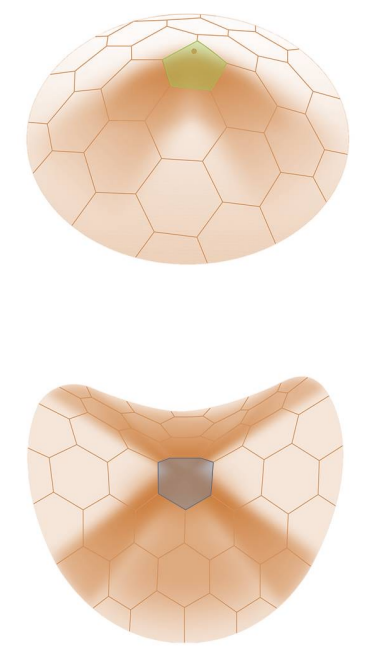

(c)

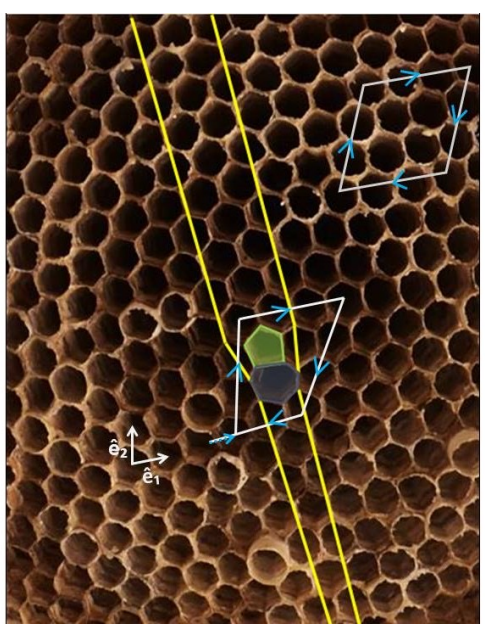

Figure 7: Illustration of emergent structure that deviates from planarity with the insertion of (a) a pentagon or (b) a heptagon. (c) A disclination pair is indicated on a wasp nest. A view of parallel lines encompassing an additional hexagonal layer as they pass through this pair, thereby leaving a scar on the lattice (dislocation line). A Burgers vector obtained from a loop enclosing the dislocation is a topological invariant. A loop not enclosing a defect is shown on the top right. $\widehat{\mathbf{e}}_{1}$ and $\widehat{\mathbf{e}}_{2}$ are the basis vectors.

of different sizes. Retaining order with cells of different sizes that are being built independently by different individuals poses a challenge, and the honeybees counter this by local sensing and introducing non-hexagonal cells where required (Nazzi 2016, Smith et al. 2021, buckling pattern in honeycomb lattices-Tarnai 1989). Similarly, from our study, we speculate that introduction of a pentagon or a heptagon adjacent to each other is possible only by a regular set of local inspections carried out by wasps to identify possible deviations from hexagonality. The angle of a regular heptagon is $128.5^{\circ}$ which is closer to that of a regular hexagon than a pentagon whose angle is $105^{\circ}$. This led us to hypothesize that heptagons could have arisen first, which were then fixed immediately by adding a pentagon. However, alternatively, the higher number of pentagons overall also suggests that moulding cells with lesser material could have erroneously resulted in pentagons that were subsequently fixed by inserting a heptagon. It is likely that experience and learning play a crucial role in repair processes. Experiments in P. fuscatus have shown the presence of an elaborate building programme that is beyond a set of linear steps, including repairing cell walls when damaged, adding pulp, and strengthening the stalk when required, which is done by multiple inspections (Downing and Jeanne 1990). This suggests that deviations from 6-sidedness are also possibly assessed via such inspections. However, it is unknown if these inspections involve identifying the number of vertices, measuring the internal angles or by Burgers vector.

Two key features that were never violated in the nests were a) convexity of individual cells and b) planar global structure. We postulate that wasps conduct systematic localized inspections to ensure that the above two features are preserved. Based on our study, we suggest that wasps detect changes in local symmetry or order and make changes to the adjoining cells to restore order. To have a planar global structure, restoration of the local order is crucial. Going further, we intend to test the role of cell size heterogeneity on the evolution and repair of topological defects. Future studies could place the nests in captivity and characterise the behaviours that precede and succeed 
the construction of cells around the defects. The introduction of defects experimentally in such captive nests would help understand the response of individuals and the group. While our study establishes the presence and repair of the defects, it is unclear if all the wasps equally participate in this process or certain keystone individuals are efficient at inspection and repair processes. These additional studies would therefore allow us to understand the behavioral mechanisms encompassing the repair of emergent topological defects.

Data availability: Data used in this manuscript will be made available upon request.

Acknowledgements: We thank Sreya Dey for taking up this idea in its pilot phase and initiating the work. Thanks to Smruti Ranjan and Abdelsalam Gena for help with data analysis. We thank Upasana Sengupta, Diana Michael, and Nageshwar Kumar for help with collecting nests.

Funding: SK acknowledges intramural funding from Ashoka University. AG thanks Science and Engineering Research Board, India (CRG/2019/003297) for fellowship. SMB thanks JC Bose Fellowship (SR/S2/JCB-71/2009) from Science and Engineering Research Board, India.

Author contributions: SK conceived and designed the study. AG and SK collected the data. SK and SMB performed and interpreted the analysis and drafted the manuscript. All authors gave final approval for publication.

Competing interests: The authors declare no competing interests.

\section{References}

Baddeley, A., Rubak, E., and Turner, R. (2015). Spatial Point Patterns: Methodology and Applications with $R$. Chapman and Hall/CRC Press, London.

Bauer, D. and Bienefeld, K. (2013). Hexagonal comb cells of honeybees are not produced via a liquid equilibrium process. Naturwissenschaften, 100(1):45-49.

Bhattacharjee, S. M. (2017). Use of Topology in physical problems. In Bhattacharjee, S. M., Mj, M., and Bandyopadhyay, A., editors, Topology and Condensed Matter Physics, pages 171-216. Springer Singapore, Singapore.

Bishop, M. and Bruin, C. (1984). The pair correlation function: A probe of molecular order. American Journal of Physics, 52(12):1106-1108.

Bonabeau, E., Theraulaz, G., Deneubourg, J. L., and Camazine, S. (1997). Self-organization in social insects. Trends in ecology $\mathcal{G}$ evolution, 12(5):188-193.

Camazine, S., Deneubourg, J., Franks, N. R., Sneyd, J., Theraulaz, G., and Bonabeau, E. (2001). Self-organization in biological systems. Princeton University Press, Princeton.

Carpenter, J. M. (1996). Phylogeny and biogeography of Polistes. In Turillazzi, S. and Eberhard, M. J. W., editors, Natural History and Evolution of Paper-Wasps, pages 18-57. Oxford University Press, Oxford, Newyork.

Ceccolini, F. (2019). New records and distribution update of Polistes (Gyrostoma) wattii Cameron, 1900 (Hymenoptera: Vespidae: Polistinae). Caucasian Entomological Bulletin, 15(2):323-326.

de Gennes, P.-G. (1979). The Physics of liquid crystals. Oxford: Clarendon Press, Oxford. 
Downing, H. and Jeanne, R. (1990). The regulation of complex building behaviour in the paper wasp, Polistes fuscatus (Insecta, Hymenoptera, Vespidae). Animal Behaviour, 39(1):105-124.

Eberhard, M. J. W. (1969). The Social Biology of Polistine Wasps. Miscellaneous Publications, Museum Of Zoology, University Of Michigan, 140:110.

Fleury, P. A. (1981). Phase Transitions, Critical Phenomena, and Instabilities. Science, 211:125-131.

Gallo, V. and Chittka, L. (2018). Cognitive Aspects of Comb-Building in the Honeybee? Frontiers in Psychology, 9:900.

Grassé, P. P. (1959). La reconstruction du nid et les coordinations interindividuelles chezBellicositermes natalensis etCubitermes sp. la théorie de la stigmergie: Essai d'interprétation du comportement des termites constructeurs. Insectes sociaux, 6(1):41-80.

Grassé, P. P. (1984). Termitology. Termite anatomy-physiology-biology-systematics. Vol. II. Colony foundation-construction. Termitology. Termite anatomy-physiology-biology-systematics. Vol. II. Colony foundation-construction. Masson, Paris.

Griffin, S. M. and Spaldin, N. A. (2017). On the relationship between topological and geometric defects. Journal of Physics: Condensed Matter, 29(34):343001.

Hales, T. C. (2001). The Honeycomb Conjecture. Discrete $\&$ Computational Geometry, 25(1):1-22.

Hansell, M. and Hansell, M. H. (2005). Animal architecture. Oxford University Press, Oxford.

Harris, W. F. (1977). Disclinations. Scientific American, 237(6):130-145.

Heggie, M. I., Haffenden, G. L., Latham, C. D., and Trevethan, T. (2016). The Stone-Wales transformation: from fullerenes to graphite, from radiation damage to heat capacity. Philosophical Transactions of the Royal Society A: Mathematical, Physical and Engineering Sciences, 374(2076):20150317.

Hepburn, H. R. and Whiffler, L. A. (1991). Construction defects define pattern and method in comb building by honeybees. Apidologie, 22(4):381-388.

Iorio, A. and Sen, S. (2007). Virus Structure: From Crick and Watson to a New Conjecture. In arXiv:0707.3690 [cond-mat, physics:math-ph, q-bio]. arXiv: 0707.3690.

Jeanne, R. L. (1975). The Adaptiveness of Social Wasp Nest Architecture. The Quarterly Review of Biology, 50(3):267-287.

Jeanne, R. L. (1979). A latitudinal gradient in rates of ant predation. Ecology, 60(6):1211-1224.

Karihaloo, B. L., Zhang, K., and Wang, J. (2013). Honeybee combs: how the circular cells transform into rounded hexagons. Journal of The Royal Society Interface, 10(86):20130299.

Karsai, I. (1999). Decentralized Control of Construction Behavior in Paper Wasps: An Overview of the Stigmergy Approach. Artificial Life, 5(2):117-136.

Karsai, I. and Pénzes, Z. (1998). Nest shapes in paper wasps: can the variability of forms be deduced from the same construction algorithm? Proceedings of the Royal Society of London. Series B: Biological Sciences, 265(1402):1261-1268. 
Karsai, I. and Pénzes, Z. (1999). Optimality of cell arrangement and rules of thumb of cell initiation in Polistes dominulus: a modeling approach. Behavioral Ecology, 11(4):387-395.

Khuong, A., Gautrais, J., Perna, A., Sbaï, C., Combe, M., Kuntz, P., Jost, C., and Theraulaz, G. (2016). Stigmergic construction and topochemical information shape ant nest architecture. Proceedings of the National Academy of Sciences, 113(5):1303-1308.

Lee, K. C., Yu, Q., and Erb, U. (2016). Mesostructure of Ordered Corneal Nano-nipple Arrays: The Role of 5-7 Coordination Defects. Scientific Reports, 6(1):28342.

Ma, J., Alfè, D., Michaelides, A., and Wang, E. (2009). Stone-Wales defects in graphene and other planar sp2 -bonded materials. Physical Review B, 80(3):033407.

Mermin, N. D. (1979). The topological theory of defects in ordered media. Reviews of Modern Physics, 51(3):591-648.

Nazzi, F. (2016). The hexagonal shape of the honeycomb cells depends on the construction behavior of bees. Scientific Reports, 6(1):28341.

Ophus, C., Shekhawat, A., Rasool, H., and Zettl, A. (2015). Large-scale experimental and theoretical study of graphene grain boundary structures. Physical Review B, 92(20):205402.

Perna, A. and Theraulaz, G. (2017). When social behaviour is moulded in clay: on growth and form of social insect nests. Journal of Experimental Biology, 220(1):83-91.

Peters, J. M., Peleg, O., and Mahadevan, L. (2019). Collective ventilation in honeybee nests. Journal of The Royal Society Interface, 16(150):20180561.

Pirk, C., Hepburn, H., Radloff, S., and Tautz, J. (2004). Honeybee combs: construction through a liquid equilibrium process? Naturwissenschaften, 91(7).

Pénzes, Z. and Karsai, I. (1993). Round shape combs produced by stigmergic scripts in social wasp. Proceedings of European Conference of Artificial Life, 93:896-905.

R Core Team (2020). R: A language and environment for statistical computing. https://www.Rproject.org/.

Reid, C. R., Lutz, M. J., Powell, S., Kao, A. B., Couzin, I. D., and Garnier, S. (2015). Army ants dynamically adjust living bridges in response to a cost-benefit trade-off. Proceedings of the National Academy of Sciences, 112(49):15113-15118.

Schilling, T., Pronk, S., Mulder, B., and Frenkel, D. (2005). Monte Carlo study of hard pentagons. Physical Review E, 71(3):036138.

Schneider, C. A., Rasband, W. S., and Eliceiri, K. W. (2012). NIH Image to ImageJ: 25 years of image analysis. Nature Methods, 9:671-675.

Seeley, T. and Heinrich, B. (1981). Regulation of temperature in the nests of social insects. John Wiley and Sons, Inc, pages 224-234.

Smith, M. L., Napp, N., and Petersen, K. H. (2021). Imperfect comb construction reveals the architectural abilities of honeybees. Proceedings of the National Academy of Sciences, 118(31):e2103605118. 
Steinhardt, P. J., Nelson, D. R., and Ronchetti, M. (1983). Bond-orientational order in liquids and glasses. Physical Review B, 28(2):784-805.

Stone, A. J. and Wales, D. J. (1986). Theoretical studies of icosahedral C60 and some related species. Chemical Physics Letters, 128(5):501-503.

Tarnai, T. (1989). Buckling patterns of shells and spherical honeycomb structures. Symmetry, pages 639-652.

Theraulaz, G. and Bonabeau, E. (1995). Coordination in Distributed Building. Science, 269(5224):686-688.

Theraulaz, G., Bonabeau, E., and Deneubourg, J. L. (1999). The mechanisms and rules of coordinated building in social insects. In Information Processing in Social Insects. Birkhäuser, Basel.

Tschinkel, W. R. (2004). The nest architecture of the Florida harvester ant, Pogonomyrmex badius. Journal of Insect Science, 4(1):21.

Tóth, L. F. (1964). What the bees know and what they do not know. Bulletin of the American Mathematical Society, 70(4):468-481.

Wenzel, J. W. (1989). Endogenous factors, external cues, and eccentric construction in Polistes annularis (Hymenoptera: Vespidae). Journal of insect behavior, 2(5):679-699.

Wenzel, J. W. (1991). Evolution of nest architecture. In Ross, K. G. and Matthews, R. W., editors, The social biology of wasps, pages 480-519. Cornell University Press, Ithaca, New York.

Zsoldos (2010). Effect of topological defects on graphene geometry and stability. Nanotechnology, Science and Applications, page 101. 